\title{
Quellen und Vorkommen kurzkettiger Alkylphenole (SCAP)
}

\author{
Steffi Fischer • Tobias Licha
}

Eingang des Beitrages: 1.6.2011 / Eingang des überarbeiteten Beitrages: 29.3.2012 / Online veröffentlicht: 6.10.2012

(C) The Author(s) 2012. Dieser Artikel ist auf Springerlink.com mit Open Access verfügbar

Zusammenfassung Aus der Literaturrecherche und eigenen Untersuchungen geht hervor, dass kurzkettige Alkylphenole (SCAP) während der Verarbeitungs- bzw. Veredelungsprozesse fester fossiler Brennstoffe ebenso in die Umwelt freigesetzt werden können, wie basierend auf Prozessen verschiedenster Industrien. Die große Bandbreite an Verwendungs- und Anwendungsmöglichkeiten von SCAP bringt viele Eintragsmöglichkeiten in die Umweltmedien mit sich. Prägnant sind die prozessbedingten SCAP-Muster (vorkommende Einzelsubstanzen) der verschiedenen Industrien und Produkte, wodurch Eintragsquellen und Fließpfade genauer quantifiziert werden können. Natürliche Eintragsquellen von SCAP mit Konzentrationen bis zu mehreren Tausend ppm sind ausschließlich Erdöle bzw. erdölführende Gesteine. Dieser Sachverhalt in Verbindung mit ihren chemisch-physikalischen Eigenschaften begründet die Verwendung der SCAP als Fluss- und Prozessindikatoren in der Erdöl-Reservoir-Erkundung und -Förderung. Anlehnend daran ist der oft noch fehlende Kenntnisstand der Umweltrelevanz und -akzeptanz von SCAP in GrundwasserSchadensfällen in Deutschland nicht nachzuvollziehen. Es wird verdeutlicht, dass SCAP neben Erdölreservoiren auch für den Wasserkreislauf als Fluss- und Prozessindikatoren prädestiniert sind.

\section{S. Fischer $(\bowtie)$}

Volkswagen AG, Postfach 1451, KSt. 4597, HK-TW/32,

34219 Baunatal, Deutschland

E-Mail: steffi.fischer@volkswagen.de

T. Licha

Angewandte Geologie, Institut für Geowissenschaften, Goldschmidtstraße 3, 37077 Göttingen, Deutschland

E-Mail: tobias.licha@geo.uni-goettingen.de

\section{Sources and occurence of short chained alkyl phenols (SCAP)}

\begin{abstract}
A literature research and own data on Short Chain Alkyl Phenols (SCAP) in the environment indicate that treatment of solid fossil fuel materials and the production of chemicals from various industries have common potenzial anthropogenic sources of SCAP compounds in the environment. Potenzial natural sources of SCAP in the environment are solely from petroliferous rocks. A wide range of SCAP applications for different industries is summarized here briefly. Many process-based inputs to the environment have been identified. Contaminations have specific "fingerprints", (unique types of SCAP compound occurrences). This enables the identification of SCAP sources. Furthermore, unique environmental properties among the variety of SCAP compounds, facilitates characterisation of contaminated sites. Knowledge of the unique SCAP "fingerprints" in conjunction with the environmentally predictable relationships of the various SCAP compounds is the basis for the use of these substances in process and flow characterisation within aquifers.
\end{abstract}

Keywords SCAP · Industrial fingerprints ·

Characterisation of groundwater pollution - Indicator for process and flow

\section{Einleitung}

„Phenole“ beschreibt eine Stoffklasse organischer Verbindungen, an deren aromatischen Kohlenwasserstoffsechsring mindestens eine Hydroxylgruppe (-OH) direkt gebunden ist (Tab. 1). Das 1834 von F. Runge unter dem Namen Karbolsäure entdeckte Phenol ist die einfachste Verbindung der 
Tab. 1 Unterklassen kurzkettiger Alkylphenole

\begin{tabular}{lllll}
\hline $\begin{array}{l}\text { Name } \\
\text { (Abkürzung) }\end{array}$ & Struktur (Beispiele) & Isomere & $\begin{array}{l}\text { Chemische } \\
\text { Formel }\end{array}$ & $\begin{array}{l}\text { Molare Masse } \\
{[\mathrm{g} / \mathrm{mol}]}\end{array}$ \\
\hline $\mathrm{C} 0 \quad$ Phenol (P) & 1 & $\mathrm{C}_{6} \mathrm{H}_{6} \mathrm{O}$ & 94,11
\end{tabular}

C1 Kresol (K)

2-Methylphenol<smiles>Cc1ccccc1O</smiles>

3

$\mathrm{C}_{7} \mathrm{H}_{8} \mathrm{O}$

108,14

\section{2,6-Dimethylphenol}<smiles>Cc1cccc(C)c1O</smiles>

6

$\mathrm{C}_{8} \mathrm{H}_{10} \mathrm{O}$

122,17

Dimethylphenol (DMP)

Ethylphenol (EP)

3-Ethylphenol

\section{$\mathrm{OH}$}<smiles>CCc1cccc(C)c1</smiles>

2,4,6-Trimethylphenol<smiles>Cc1cc(C)c(O)c(C)c1</smiles>

3-Propylphenol<smiles>CCCc1cccc(O)c1</smiles>

2-Isopropylphenol

Propylphenol (PP)<smiles>CC(C)c1ccccc1O</smiles>

Isopropylphenol (iPP)
6

$\mathrm{C}_{9} \mathrm{H}_{12} \mathrm{O}$

136,20
3 
Abb. 1 Prägnante Eigenschaften umweltrelevanter Phenole

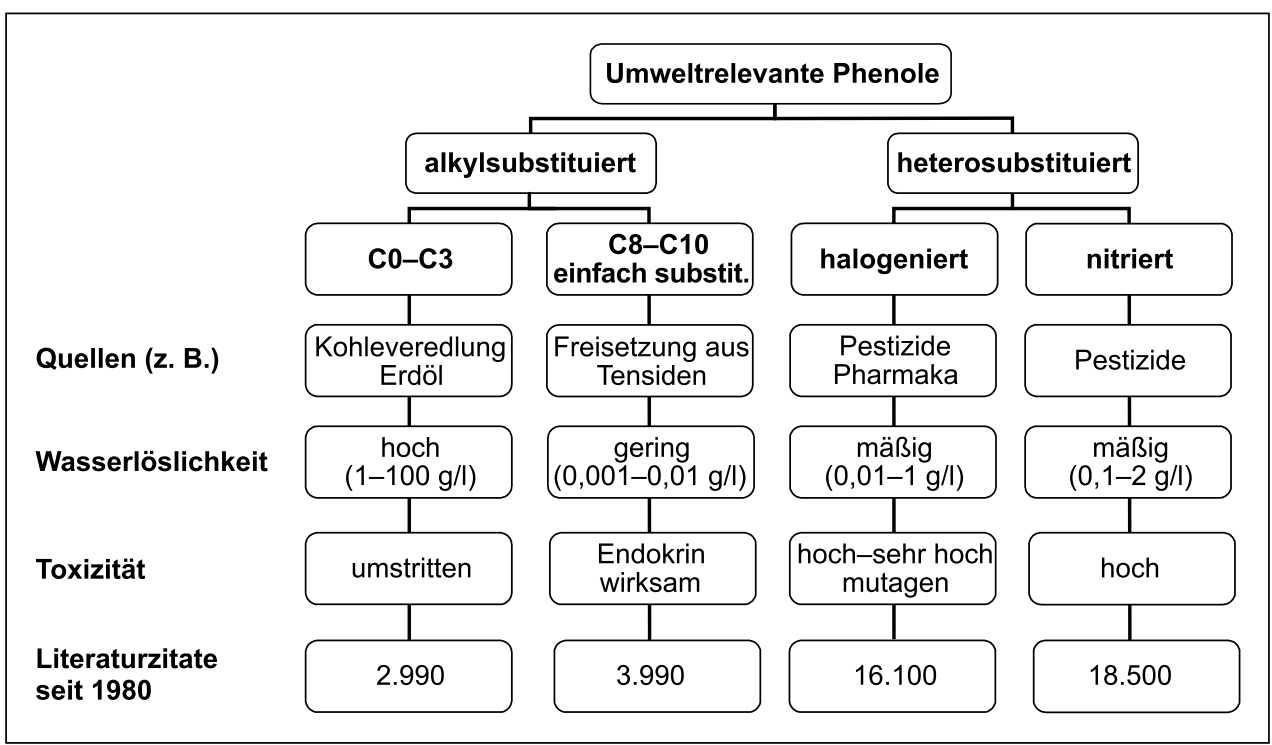

Stoffklasse und besteht aus einem Benzenring mit einer Hydroxylgruppe. Die Anzahl der Hydroxylgruppen und damit die Zahl der donierbaren Protonen bestimmt in Analogie zu Säuren die Wertigkeit von Phenolen. Allerdings nimmt mit steigender Anzahl an Hydroxylgruppen die Stabilität der Verbindungen drastisch ab (Isacchi et al. 2007). Neben den „freien“ Phenolen existieren die Phenolderivate, bei denen Substituenten das Wasserstoffatom an der Hydroxylgruppe ersetzen. Diese Derivate besitzen oft nur dann eine Umweltrelevanz, wenn sich aus ihnen durch Reaktionen (z. B. Hydrolyse) die entsprechenden Phenole wieder freisetzen. Beispielhaft dafür stehen Alkylphenolethoxylate (Etherderivate, Einsatz z. B. in Kosmetika und Waschmitteln), aus welchen durch Abbau endokrin wirksame Phenole mit entsprechender Umweltrelevanz freigesetzt werden (Giger et al. 1984, Brunner et al. 1988).

Aufgrund der großen Variationsbreite an Substituenten sowie deren Positionen am aromatischen Ring beinhaltet die Stoffgruppe „Phenole“ eine große Bandbreite an Verbindungen mit breitem Eigenschaftsspektrum. Man unterscheidet dabei zwischen kohlenstoffhaltigen und nicht kohlenstoffhaltigen Substituenten bzw. zwischen alkylsubstituierten und heterosubstituierten Phenolen (Abb. 1). Dabei werden in letzterer Gruppe die toxischen Chlor- und Nitrophenole zusammengefasst (z. B. Pentachlorphenol), deren Relevanz und Umweltverhalten weitestgehend geklärt und geregelt sind. Dies spiegelt sich u. a. in der Anzahl der vorhandenen Literaturzitate wider (z. B. Fischer et al. 2000, Shiu et al. 1994, Abb. 1). Im Gegensatz dazu zeigen die alkylsubstituierten Phenole, insbesondere die kurzkettigen $\left(\mathrm{C}_{0}-\mathrm{C}_{3}\right)$ mit nur knapp 3.000 Zitaten und umstrittener Toxizität erhöhten Forschungsbedarf auf.

Seit einigen Jahren nehmen die Alkylphenole i. A. im Wasserkreislauf einen wachsenden Stellenwert in der Umweltforschung ein. Dies trifft vor allem auf die schlecht wasserlösliche aber endokrin wirksame Fraktion zu (z. B. Nonylphenol), teilweise auch auf die gut wasserlösliche Fraktion, die der kurzkettigen Alkylphenole (z. B. Phenol: $102,1 \mathrm{~g} / \mathrm{l}$ bei $25{ }^{\circ} \mathrm{C}$, Varhanickova et al. 1995). Letzterer kommt gerade wegen ihrer hohen Mobilität in Grundwasserleitern eine besondere Relevanz zu, welche im Wesentlichen auf geringe Octanol-Wasser-Verteilungskoeffizienten basieren (z. B. Phenol: $\log K_{\mathrm{OW}} 1,46$, Licha 2002). Zusätzlich sei angemerkt, dass Alkylphenole zu den am meisten hergestellten Chemikalien gehören und somit unter den Top 50 der USA zu finden sind (Ahmaruzzaman 2008). Beispielsweise werden in Deutschland jährlich mehr als 30.000 t Kreosot hergestellt (Böhm et al. 2002).

Die Gruppe der Alkylphenole beinhaltet Phenolhomologe, bei denen mindestens ein Wasserstoffatom am Benzenring durch eine Alkylgruppe substituiert ist (Tab. 1). Durch die große Variationsbreite im Hinblick auf die möglichen kohlenstoffhaltigen Substituenten, deren Anzahl, Stellungen am Benzenring, sowie deren Kettenlängen und Isomerie existieren eine Vielzahl von Einzelverbindungen mit breitem chemisch-physikalischem Eigenschaftsspektrum. In Relation zu anderen Schadstoffgruppen sind diese untereinander zwar als ähnlich anzusehen, allerdings innerhalb der SCAP ausgeprägt genug um relevante Unterschiede festlegen zu können. Aufgrund dessen wurde die Gruppe der Alkylphenole von Licha (2002) nach ihren Eigenschaften und Vorkommen weiter unterteilt. Dabei wurde vorgeschlagen, die kurzkettigen Alkylphenole (SCAP - short chained alkylphenols), zu denen die alkylierten Phenolhomologe mit bis zu 3 Kohlenstoffatomen außerhalb des Benzenrings gehören, in einer eigenen Gruppe mit ähnlichen physiko-chemischen Eigenschaften der Einzelverbindungen sowie gemeinsamen Eintragsquellen/Vorkommen zusammenzufassen. Die Gruppe der SCAP $\left(\mathrm{C}_{0}-\mathrm{C}_{3}\right)$ bzw. kurzkettigen Alkylphenole beinhaltet inklusive des Phenols 35 
Einzelverbindungen mit variabler Anzahl an Isomeren pro Substanzklasse (Tab. 1).

Während Schadstoffklassen mit unpolaren Verbindungen wie z. B. PAK (polyzyklische aromatische Kohlenwasserstoffe) oder CKW (chlorierte Kohlenwasserstoffe) einen weitaus höheren Kenntnisstand hinsichtlich der Stoffeigenschaften und somit auch der Umweltrelevanz aufgrund von wissenschaftlichen Studien aufweisen, zeigen sich bei den polaren Schadstoffklassen teilweise große Wissenslücken hinsichtlich ihres Umweltverhaltens und somit auch hinsichtlich der Beurteilung von Schadensfällen. Verdeutlicht wird dieser Sachverhalt durch wenig bis keine Betrachtung und Bewertung von SCAP bei Grundwasserschadensfällen und deren Sanierungen auf andere Schadstoffgruppen mit potenziellen Gehalten an SCAP; sowohl von Seiten der Behörden als auch von Sachverständigen. Umso erstaunlicher erscheint die hohe Relevanz von SCAP in der Erdölindustrie, speziell der Rohöl-Förderung und Reservoir-Erkundung. Zum einen muss aufgrund des hohen Umweltgefährdungspotenziales die Behandlung anfallender Prozesswässer während der Erdölförderung auf die SCAPKonzentrationen abgestimmt sein (Bennett et al. 2007), zum anderen werden diese Substanzen zur Erkundung als quantitative Tracer z. B. für das Fließverhalten (Migration) herangezogen (Larter \& Bennett 2011). In diesem Artikel sind die Quellen und das Vorkommen kurzkettiger Alkylphenole zusammenfassend mit dem Ziel dargestellt, neben einer besseren Quellzuordnung auch zu einem tieferen Verständnis dieser Stoffgruppe beizutragen. Darüber hinaus soll das Anwendungspotenzial der SCAP als Fluss- und Prozessindikatoren für den Wasserkreislauf verdeutlicht werden. Die Ergebnisse basieren neben einer Literaturauswertung auch auf eigenen Arbeiten der Autoren zu dieser Thematik.

\section{Chemische Analytik von kurzkettigen Alkylphenolen in Boden und Wasserproben}

Die im Rahmen dieser Studie selbst bestimmten Konzentrationen an SCAP wurden mithilfe einer dafür entwickelten chemischen Analysenmethode ermittelt. Diese hier beschriebene Methode kann alle relevanten SCAP-Isomere erstmals vollständig trennen, wodurch es keine Verwechslung von Isomeren als auch falsch positive Ergebnisse geben kann.

Bei Bestimmung aus Feststoffen wurde eine Mikrowellenextraktion für $30 \mathrm{~min}$ bei $100{ }^{\circ} \mathrm{C}$ mit originalfeuchter Probe und methanolischer $\mathrm{NaOH}$ durchgeführt. Zur weiteren Bearbeitung wurde der pH-Wert des Extraktes auf 79 eingestellt. Wässrige Proben wurden entsprechend dem Arbeitsbereich mit Blindwert-freiem Wasser verdünnt.

Die Derivatisierung der Analyten erfolgte aus Karbonatgepufferter Lösung mit Essigsäureanhydrit im Ultraschall- bad. Die entstandenen Acetate wurden mit einem Hexanähnlichem Lösemittel extrahiert und die organische Phase anschließend über $\mathrm{Na}_{2} \mathrm{SO}_{4}$ getrocknet.

Die Messung der Analyten erfolgte mittels eines Gaschromatographen (Agilent Technologies, Modell 6890) mit anschließender massenspektrometrischer Detektion im SIMModus (Agilent Technologies, Modell 5972A). Die acetylierten Phenolisomere konnten auf einer polaren WAXSäule (VF-WAXms, Agilent) vollständig getrennt werden.

Die Qualität der Ergebnisse wurde über interne Standards (2-Fluorphenol, 4-Fluor-o-Kresol, 2,4 DMP d ${ }^{3}$ ) abgesichert. Zusätzlich wurde intern und extern kalibriert.

\section{Vorkommen und Quellen kurzkettiger Alkylphenole}

Phenole im Allgemeinen sind wichtige chemische Grundund Wirkstoffe und haben daher ein breites Einsatzspektrum. Direkte Verwendung finden Phenole heutzutage in freier oder derivatisierter Form als Produktbestandteile wie z. B. Stabilisatoren, Weichmacher und Öladditive; ebenso während verschiedener Herstellungsprozesse z. B. von Kosmetik, Geschmacks- und Aromastoffen in Lebensmitteln, Sprengstoffen, Kunstfasern, Polymeren und Tensiden. Darüber hinaus setzen viele Branchen phenol- und phenolderivathaltige Zubereitungen ein, z. B. Holz-, Farb- und v. A. metallverarbeitende Industrien. Eigene Analysen von Emulsion aus einer metallverarbeitenden Produktion zeigen SCAP-Konzentrationen bis knapp $9 \mathrm{mg} / \mathrm{l}$ in der Summe (Tab. 5), mit breiter Isomerenverteilung.

Im Gegensatz zu den freien Phenolen existieren für Phenolderivate auch einige natürliche Eintragsquellen, da sie Bestandteile von z. B. Pflanzen (Kollmansberger 2007) sind. Diese Derivate sind nur dann umweltrelevant, wenn aus ihnen entsprechend toxische freie Phenole entstehen können.

Die einzige relevante, im Hinblick auf Konzentration und Bandbreite, natürliche Eintragsquelle kurzkettiger Alkylphenole sind Erdöle bzw. erdölführende Gesteine. Mit Ausnahme des 3-n-Propylphenols sind alle SCAP in verschiedenen Erdölen nachgewiesen (Ippolo et al. 1992, Bennett et al. 1996, 2007, Bastow et al. 2003, Taylor et al. 1997). Trotz stark variierender SCAP-Konzentrationen zwischen keinen und mehreren 10.000 ppm in verschiedensten Rohölen (unterschiedliche Entstehungsorte und -zeiten, Ursprungsmaterialien und Alterierungsgrade), zeigen alle Rohöle einer Versuchsreihe äquivalente Isomerenverteilungen unter den $\mathrm{C}_{0}$ $\mathrm{C}_{2}$ Isomeren; lediglich bei den $\mathrm{C}_{3}$-Isomeren zeigten sich Unterschiede im Phenolmuster (Taylor et al. 1997). SCAP werden als effektive Indikatoren für Migrationsprozesse von Erdölen (Larter et al. 1996) beschrieben. Des Weiteren können sie die Interaktionen zwischen Fluiden und Gesteinen, sowie die Benetzbarkeiten des Systems beeinflussen (Bennett et al. 2007, Larter et al. 2007). Die Literaturrecherche 
Tab. 2 Prozessbasierte SCAP, verändert nach Ullmann's Encyclopedia (1995), Stoffdatenbanken des UBA (Umweltbundesamt 2009); ,j“ = ja; „," = nein; () keine eindeutige Bewertung

\begin{tabular}{|c|c|c|c|c|c|c|c|c|}
\hline & \multicolumn{2}{|c|}{$\begin{array}{l}\text { Kohle- und } \\
\text { Öl-Sumpfphasehydrierung }\end{array}$} & \multicolumn{3}{|c|}{ Verschwelen (niedrige Temperaturen) } & \multicolumn{3}{|c|}{ Verkoken (hohe Temperaturen) } \\
\hline & Steinkohle & Braunkohle & Steinkohle & Braunkohle & Torf & Steinkohle & Torf & Braunkohle \\
\hline Phenol & $\mathrm{j}$ & (j) & $\mathrm{j}$ & (j) & $\mathrm{j}$ & (n) & (j) & (j) \\
\hline Kresole & $\mathrm{j}$ & (j) & $\mathrm{j}$ & (j) & $\mathrm{j}$ & $\mathrm{j}$ & (j) & (j) \\
\hline 2,4-DMP & & & & & & $\mathrm{n}$ & & \\
\hline \multicolumn{9}{|l|}{ 2,6-DMP } \\
\hline \multicolumn{9}{|l|}{ 3,4-DMP } \\
\hline 3.5-DMP & $\mathrm{j}$ & (j) & $\mathrm{j}$ & (j) & $\mathrm{j}$ & $\mathrm{j}$ & (j) & (j) \\
\hline \multicolumn{9}{|l|}{ 2,5-DMP } \\
\hline 2,3-DMP & & & & & & $\mathrm{n}$ & & \\
\hline Ethylphenole & (j) & (j) & $\mathrm{j}$ & (n) & $\mathrm{n}$ & $\mathrm{n}$ & (n) & (n) \\
\hline 2,4,6-TMP & (j) & (j) & $\mathrm{n}$ & (n) & $\mathrm{j}$ & $\mathrm{n}$ & (n) & (n) \\
\hline restl. TMP & $\mathrm{n}$ & $\mathrm{n}$ & $\mathrm{n}$ & $\mathrm{n}$ & & $\mathrm{n}$ & $\mathrm{n}$ & $\mathrm{n}$ \\
\hline iPP & $\mathrm{n}$ & $\mathrm{n}$ & $\mathrm{n}$ & $\mathrm{n}$ & $\mathrm{n}$ & $\mathrm{n}$ & $\mathrm{n}$ & $\mathrm{n}$ \\
\hline nPP & $\mathrm{n}$ & $\mathrm{n}$ & $\mathrm{n}$ & $\mathrm{n}$ & $\mathrm{n}$ & $\mathrm{n}$ & $\mathrm{n}$ & $\mathrm{n}$ \\
\hline 3-E-5-MP & $\mathrm{n}$ & $\mathrm{n}$ & $\mathrm{n}$ & $\mathrm{n}$ & $\mathrm{n}$ & $\mathrm{n}$ & $\mathrm{n}$ & $\mathrm{n}$ \\
\hline
\end{tabular}

zeigt, dass in der Erdöl-Industrie bzw. im Erdöl-ReservoirSystem SCAP bereits erfolgreich als Fluss- und Prozessindikatoren Verwendung finden (z. B. Bennett et al. 2007, Larter \& Bennett 2011, Taylor et al. 1997).

Eine unbedeutende, aber zu erwähnende ,natürliche“ Eintragsquelle von SCAP sind flüssige heterotrophe Stoffwechselendprodukte. Während menschliche Ausscheidungen hauptsächlich Phenol und Kresole enthalten, kann tierische Gülle zusätzlich deutliche Konzentrationen an Dimethylphenolen aufweisen (Lo et al. 2008). Während für menschliche Ausscheidungen aufgrund des Abwassersystems eine geringe Wahrscheinlichkeit für die Einbringung dieser Substanzen in die Umwelt besteht, ist der Eintrag über Gülle durchaus zu beachten.

Um die Quellen kurzkettiger Alkylphenole spezifizieren zu können und damit ganz bestimmte Kontaminationsmuster an SCAP mit entsprechenden Eintragsquellen assoziieren zu können, sind in den Tabellen 2 und 3 die relevantesten Kontaminationsquellen hinsichtlich der Vorkommen einzelner Phenole weiter aufgeschlüsselt, soweit diese aus Literaturdaten eindeutig zugänglich waren.

In Tabelle 2 sind Beispiele prozessbasierter Eintragsquellen von SCAP verschiedener Ausgangsmaterialien zusammengestellt. Bei der Verarbeitung bzw. Veredelung von Kohlen zu Teer, Koks, Pech usw. werden hohe Temperaturen benötigt um dem Endprodukt unerwünschte Bestandteile wie Bitumen, Wasser, Sauerstoff und Stickstoff abzutrennen. Im Wesentlichen wird unterschieden zwischen Verschwelen und Verkoken; während bei der Verschwelung als unvollständige Verbrennung Temperaturen zwischen 500 und $600{ }^{\circ} \mathrm{C}$ erreicht werden, liegen diese bei der Verkokung bei 1.000 bis $1.400{ }^{\circ} \mathrm{C}$ (Gundermann 1964). Das dem Ausgangsmaterial ausgetriebene Rohgas mittels beider Verfahren enthält u. a. Ammoniak, Schwefelwasserstoff, Benzol, Naphthalin und Phenole. Somit können sowohl bei der Verschwelung als auch bei der Verkokung von Kohlen prozessbedingt Abwässer mit nennenswerten Gehalten an kurzkettigen Alkylphenolen anfallen.

Wenn auch an dieser Stelle nicht wirklich umweltrelevant, so ist die unbeabsichtigte und im oberen $\mu \mathrm{g} / \mathrm{l}$ Bereich anzutreffende Konzentration an 4-Ethylphenol (Tab. 3) in einigen Rotweinen erwähnenswert (Rayne \& Eggers 2007), ebenso in nativen Olivenölen (Brenes et al. 2004). Auch eigene Untersuchungen zeigen 4-EP mit $>2 \mathrm{mg} / \mathrm{l}$ als dominierende Substanz unter den SCAP z. B. im Calvados (Tab. 5). Dieses Phenol entsteht durch die Aktivität bestimmter Pilze während der Reifung und gibt dem Wein eine unangenehme Note nach „Pferdeschweiß“ oder Leder. Neben einer geschmacklichen Beeinträchtigung werden auch gesundheitliche Aspekte dieser Begleitkomponente aktuell diskutiert (Rayne \& Eggers 2007).

In Gehölzen und fossilen Brennstoffen, wie Braunkohle, Torf oder Steinkohle sind keine SCAP direkt enthalten. Diese entstehen erst bei thermischer Zersetzung organischer Polymere, d. h. bei der Verarbeitung bzw. Veredelung dieser fossilen Brennstoffe. Auch bei Waldbränden kommt es zu thermischer Zersetzung von organischen Polymeren und somit zur Entstehung bzw. Freisetzung kurzkettiger Alkylphenole. Ein Kuriosum dieser thermischen Zersetzung wird bei der Herstellung bestimmter Whiskeysorten (Malt) aus Schottland bewusst ausgenutzt. Im Herstellungsprozess wird die vollständige Trocknung von Grünmalz bei 


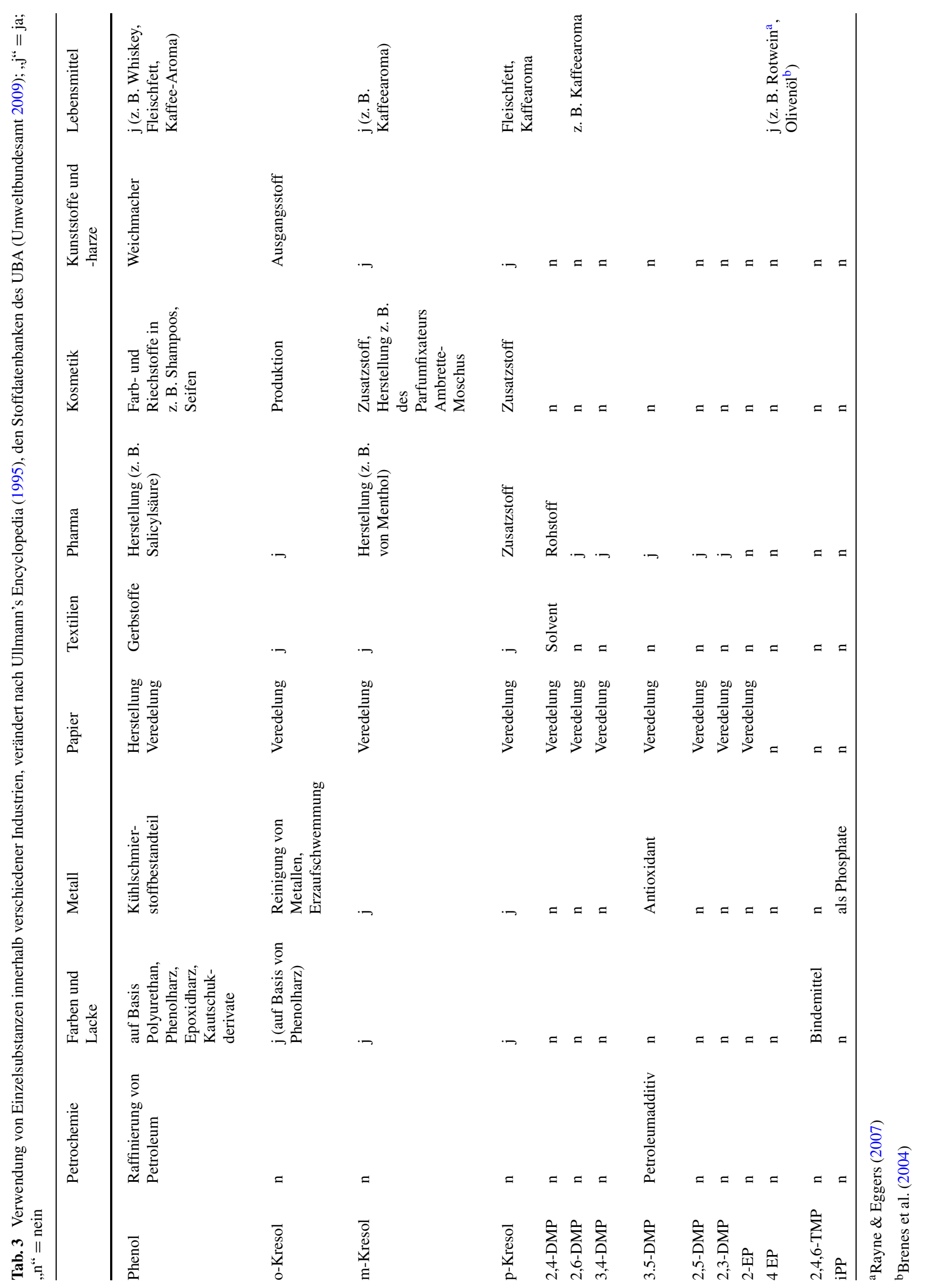


einigen Sorten über Torffeuer realisiert. Dieser Torf ist bedingt durch das Klima mit einer Restfeuchte behaftet und schwelt daher, was erhebliche Mengen an SCAP freisetzt. Das resultierende sehr rauchig-aromatische Getränk enthält daher eine beachtliche Phenolkonzentration. Eigene Untersuchungen zeigen SCAP-Konzentrationen dieser Whiskeysorten bis zu $23 \mathrm{mg} / \mathrm{l}$ (Tab. 5). Allein die Lagerungszeit (Alter des Whiskeys) wirkte sich positiv auf die Phenolkonzentration aus. Vermutlich bedingt durch Oxidationsprozesse, sinkt während der Lagerung die Phenolkonzentration messbar ab. Andere Malt-Whiskey-Hersteller hingegen setzen bewusst auf Herstellungsprozesse, bei denen die Konzentrationen von SCAP mit ca. $1 \mathrm{mg} / \mathrm{l}$ im Endprodukt nicht überschritten werden, wie in Tabelle 5 beispielhaft an einem europäischen Malt Whiskey gezeigt ist.

Die Ergebnisse eines hochwertigen Cognacs aus Tabelle 5 stehen beispielhaft für eine Reihe verschiedenster Alkohole bei denen SCAP in sehr geringen Konzentrationen gefunden wurden. Bei diesen Untersuchungsreihen waren Phenol, p-Kresol und 2-Ethylphenol die am häufigsten nachzuweisenden Isomere.

Nach Kolbe (2008) ist die Art des Entschwefelungsprozesses (Veredelung von Destillationsfraktion) bei Heizölen und Diesel-Kraftstoffen für die Gesamtkonzentration und das Isomeren-Muster an SCAP maßgeblich entscheidend. So konnte im Zuge der Arbeit festgestellt werden, dass sich die Phenolgehalte bei der klassischen Hydrodesulfurierung von Heizöl und Diesel proportional zu den Schwefelgehalten verhalten; hingegen bei der Anwendung eines neu entwickelten Entschwefelungsverfahrens die Gehalte an SCAP nur unwesentlich verändert wurden. Interessant ist auch die Feststellung, dass es während der klassischen Entschwefelung zu vermehrtem Abbau höher alkylierter Phenole kommt, bei den SCAPs besonders der ortho- $\mathrm{C}_{2}$ - und ortho- $\mathrm{C}_{3}$-Substanzen. Somit erklären sich z. B. die differenten Phenolmuster-Ergebnisse vor und nach der Entschwefelung bei dem verwendeten spanischen Diesel. Während vor der Entschwefelung die $\mathrm{C}_{2}$ - und $\mathrm{C}_{3}$-Isomere innerhalb der SCAP dominieren, zeigt sich nach der Entschwefelung eine Dominanz von Phenol und Kresolen (Kolbe 2008).

Nicht nur bei der fossilen Brennstoffverarbeitung sondern auch bei der Weiterverarbeitung der wirtschaftlich interessanten Alkylphenole können belastete Abwässer anfallen. Hierbei sei angemerkt, dass die Weiterverarbeitung meist von der chemischen Industrie selbst und nicht in den Kokereien stattfindet, wodurch weitere Eintragspfade von SCAP in die Umwelt möglich sind. In Tabelle 3 sind verschiedene Industriezweige zusammengestellt, die Einzelsubstanzen aus der Gruppe der SCAP zu unterschiedlichen Produkten weiterverarbeiten, teilweise als Rohstoff, als $\mathrm{Zu}$ satzstoff oder als Additive. Während das Phenol und die Kresole in fast allen aufgeführten Industriezweigen aus Tabelle 3 Verwendung finden, wird die Zuordnung der restli- chen Einzelsubstanzen aus der Gruppe der kurzkettigen Alkylphenole spezifischer. Beispielhaft dafür seien Isopropylphenole genannt. Aus eigenen Untersuchungen (Tab. 5) geht hervor, dass einige Öle, besonders temperaturbeständige Hydrauliköle, Phosphorsäureester auf Isopropylphenolbasis beinhal(te)ten, wobei das Phosphat als Säure mit den Hydroxylgruppen verschiedener Isopropylphenolisomere verestert ist. Diese Ester hydrolysieren im Kontakt mit Wasser und setzen dadurch Isopropylphenole frei. In eigenen Untersuchungen bestanden diese Ester z. B. in einem Hydrauliköl aus einer metallverarbeitenden Industrie neben Phenol $(72 \%)$ vorwiegend aus dem 2- (15\%), 4- (10\%) und 3-Isopropylphenolen (2\%). In den vermutlich dazugehörigen Grundwasserschadensfällen mit Altern zwischen 10 und 50 Jahren war allerdings 2-Isopropylphenol in Bereichen von 1 bis $5 \mathrm{mg} / \mathrm{l}$ die dominierende Verbindung unter den kurzkettigen Alkylphenolen (Tab. 5). Überwiegen die Konzentrationen von Isopropylphenolen in wässrigen Proben signifikant gegenüber den restlichen SCAP-Isomeren konnte anhand der eigenen Untersuchungen festgestellt werden, dass es sich beim Verursacher der Grundwasserkontamination um metallverarbeitende Industrie handeln bzw. gehandelt haben muss.

In Tabelle 4 sind einige Produktklassen zusammengeführt, die ganz bestimmte Substanzen der SCAP-Gruppe enthalten. Werden diese Produkte angewendet, kann es zu Einträgen in die Umwelt kommen. Während z. B. das $\mathrm{m}$-Kresol als einziger Vertreter ein Bestandteil des photographischen Entwicklers ist, enthalten Pflanzenschutzmittel auch andere Verbindungen aus der Gruppe. Die größte Bandbreite an SCAP zeigen bzw. zeigten phenolhaltige Desinfektionsmittel, wobei hier angemerkt sei, dass sie aufgrund ihrer Toxizität und Nebenwirkungen in der Medizin in Deutschland heute nur mehr begrenzt eingesetzt werden (Wiedenmann 2011). Phenol- und/oder Kresol-haltige Desinfektionsmittel für den Hausgebrauch und kommerziellen Einsatz sind allerdings weiterhin erhältlich (Emsley 2009).

Eine weitere bedeutende Quelle für kurzkettige Alkylphenole sind Treibstoffe, in welchen SCAP als Additive mit der Funktion als Schmier- und Konservierungsmittel enthalten sind. Mit wenigen Ausnahmen enthalten Benzine mit bis zu 180 mg/l höhere Konzentrationen an SCAP als Diesel mit maximal ca. $31 \mathrm{mg} / \mathrm{l}$ (Wasinski 2004). Ein additivfreies Benzin mit nur knapp 0,5 mg/l SCAP in der Summe belegt, dass Phenole nicht Bestandteil der entsprechenden Destillationsfraktionen - auch wenn diese Phenole im Erdöl vorkommen - sondern echte Additive in Analogie zu z. B. Methyltertiärbutylether (MTBE) sind. Die Phenole sind allerdings im Vergleich zu MTBE in deutlich geringeren Konzentrationen zugesetzt. Anders verhält es sich in der Destillationsfraktion von Diesel. Ein additivfreier Diesel-Kraftstoff enthält bis auf 2,5-DMP alle Substanzen in ähnlich hohen Konzentrationen wie ein Diesel mit Additiven (Wasinski 2004), 
Tab. 4 Anwendung von Einzelsubstanzen verschiedener Industriezweige, verändert nach Ullmann's Encyclopedia (1995), Stoffdatenbanken des UBA (Umweltbundesamt 2009); , j“ = ja; ,,n“ = nein

\begin{tabular}{|c|c|c|c|c|}
\hline & Pflanzenschutzmittel & Photographie & Desinfektionsmittel & Explosivstoffe \\
\hline Phenol & Insektizide, Herbizide & $\mathrm{n}$ & Ausgangsstoff (Veterinärmedizin) & $\mathrm{j}$ \\
\hline o-Kresol & Herbizide & $\mathrm{n}$ & Produktion & Produktion \\
\hline m-Kresol & Pestizide allgemein & Photographischer Entwickler & Zusatzstoff & Zusatzstoff \\
\hline p-Kresol & $\mathrm{n}$ & $\mathrm{n}$ & $\mathrm{j}$ & $\mathrm{n}$ \\
\hline 2,4-DMP & $\mathrm{n}$ & $\mathrm{n}$ & $\mathrm{j}$ & $\mathrm{n}$ \\
\hline 2,6-DMP & $\mathrm{n}$ & $\mathrm{n}$ & $\mathrm{j}$ & $\mathrm{n}$ \\
\hline 3,4-DMP & $\mathrm{n}$ & $\mathrm{n}$ & $\mathrm{j}$ & $\mathrm{n}$ \\
\hline 3.5-DMP & Insektizide, Fungizide & $\mathrm{n}$ & Ausgangs- bzw. Zusatzstoff & $\mathrm{n}$ \\
\hline 2,5-DMP & $\mathrm{n}$ & $\mathrm{n}$ & $\mathrm{j}$ & $\mathrm{n}$ \\
\hline 2,3-DMP & $\mathrm{n}$ & $\mathrm{n}$ & Ausgangs- bzw. Zusatzstoff & $\mathrm{n}$ \\
\hline 2-EP & $\mathrm{n}$ & $\mathrm{n}$ & $\mathrm{n}$ & $\mathrm{n}$ \\
\hline 2,4,6-TMP & $\mathrm{n}$ & $\mathrm{n}$ & $\mathrm{n}$ & $\mathrm{n}$ \\
\hline iPP & $\mathrm{n}$ & $\mathrm{n}$ & $\mathrm{n}$ & $\mathrm{n}$ \\
\hline
\end{tabular}

was darauf hindeutet, dass sie bereits in der entsprechenden Destillationsfraktion enthalten sind. Unterschiedliche Siedetemperaturen während der fraktionierten Kondensation von Erdöl spiegeln sich in den Isomerenverteilungen der SCAP in Benzinen (70-180 $\left.{ }^{\circ} \mathrm{C}\right)$ und Diesel $\left(240-300{ }^{\circ} \mathrm{C}\right)$ wider. In Benzinen und im Zweitaktgemisch dominieren i.d.R. das Phenol und die Kresole, während bei den Dieseltreibstoffen eher die höher siedenden Substanzen wie DMP und TMP zu finden sind. Bedingt durch diese Tatsache wurde das Verhältnis von DMP zu Phenolen und Kresolen erstmals von Laespada et al. (1999) verwendet und von Wasinski (2004) für dessen Vergleiche von Treibstoffen aufgegriffen. Offensichtlich spiegelt ein Verhältnis von $\leq 1$ ein Benzin bzw. ein Zweitaktgemisch wider, während Verhältnisse $>2$ eindeutig auf Diesel hinweisen. Aufgrund dieses Unterscheidungsmerkmales ist die Eignung von SCAP als Indikatoren zur Identifikation eines Treibstoffes (somit auch eines Treibstoff-Schadensfalles) näher zu prüfen. Diese Aussage wird z. B. auch von Wasinski (2004) bestätigt, indem er aufgrund einer Korrelationsanalyse das sog. Alkylphenolmuster von Treibstoffen und das daraus resultierende Potenzial zur Identifizierung von Treibstoffen beschreibt.

Eigene Untersuchungen von Phenolkontaminationen in Grundwasser der letzten 8 Jahre umfassen insgesamt 16 Grundwasserschadensfälle, wobei SCAP an 15 Standorten in signifikanten Konzentrationen bis in den mittleren $\mathrm{mg} / \mathrm{l}$ Bereich angetroffen wurden, obwohl ein Großteil der Schadensfälle 60 Jahre und älter waren. Die Bandbreite an Prozessen und Ausgangsstoffen, die zu Grundwasserschadensfällen mit kurzkettigen Alkylphenolen führen, sind vielfältig. Eine vorsichtige Schätzung der Autoren für solche Schadensfälle ergibt mindestens 3.000 potenzielle Fälle in der EU. Der Negativbefund eigens untersuchter Grundwasserschadensfälle bezieht sich auf 3 einzelne Proben eines
Standortes, auf dem Kohle veredelt wurde. Ob ein ursächlicher Zusammenhang zwischen Negativbefund und Zeit, Ort sowie Art der Probennahme bestand, konnte abschließend für jenen Standort nicht geklärt werden. Es liegt aufgrund der Erfahrung von anderen Standorten jedoch die Vermutung nahe, dass Zeit und Ort für die exakte Kartierung der Phenolfahne von entscheidender Bedeutung sind. Aufgrund des äußerst dynamischen Transportverhalten von SCAP können bei hoch durchlässigen Sedimenten Konzentrationsschwankungen von bis zu 3 Größenordnungen binnen weniger Monate an einigen Brunnen auftreten (Licha et al. 2003). Diese Effekte sind unbedingt bei Planungen von Stichtagsmessungen und Sanierungsanlagen (z. B. Art und Kapazität von Abwasservorbehandlungen) sowie bei der Beurteilung von Sanierungserfolgen zu beachten.

Die Anwesenheit nur eines Vertreters aus der Gruppe der kurzkettigen Alkylphenole in realen Proben ist eine absolute Rarität. Nur weniger als $1 \%$ aller eigenen Analysen wies weniger als zwei SCAP auf. Eine deutliche Ausnahme bildet hier wässriges Bodeneluat, welches zwischen 2 und $10 \mu \mathrm{g} / \mathrm{l}$ Phenol, und zwar nur das substituentenfreie Phenol, enthalten kann. Mehrere Böden wurden dazu in eigenen Arbeiten untersucht und in allen Eluaten konnte Phenol nachgewiesen werden. Zur Überprüfung, ob Phenol in freier Form in den Böden bereits vorlag, wurden diese Böden zusätzlich mit Methanol extrahiert. Da in diesen Alkoholauszügen kein Phenol nachgewiesen werden konnte, musste geschlussfolgert werden, dass die Böden initial frei von Phenol waren und erst im Kontakt mit Wasser durch vermutlich mikrobiologische Aktivitäten Phenol freigesetzt wurde. Auch wenn diese Mengen gering sind, ist diese ,natürliche' Quelle bei der Beurteilung von z. B. Sickerwässern jedoch zu beachten. 


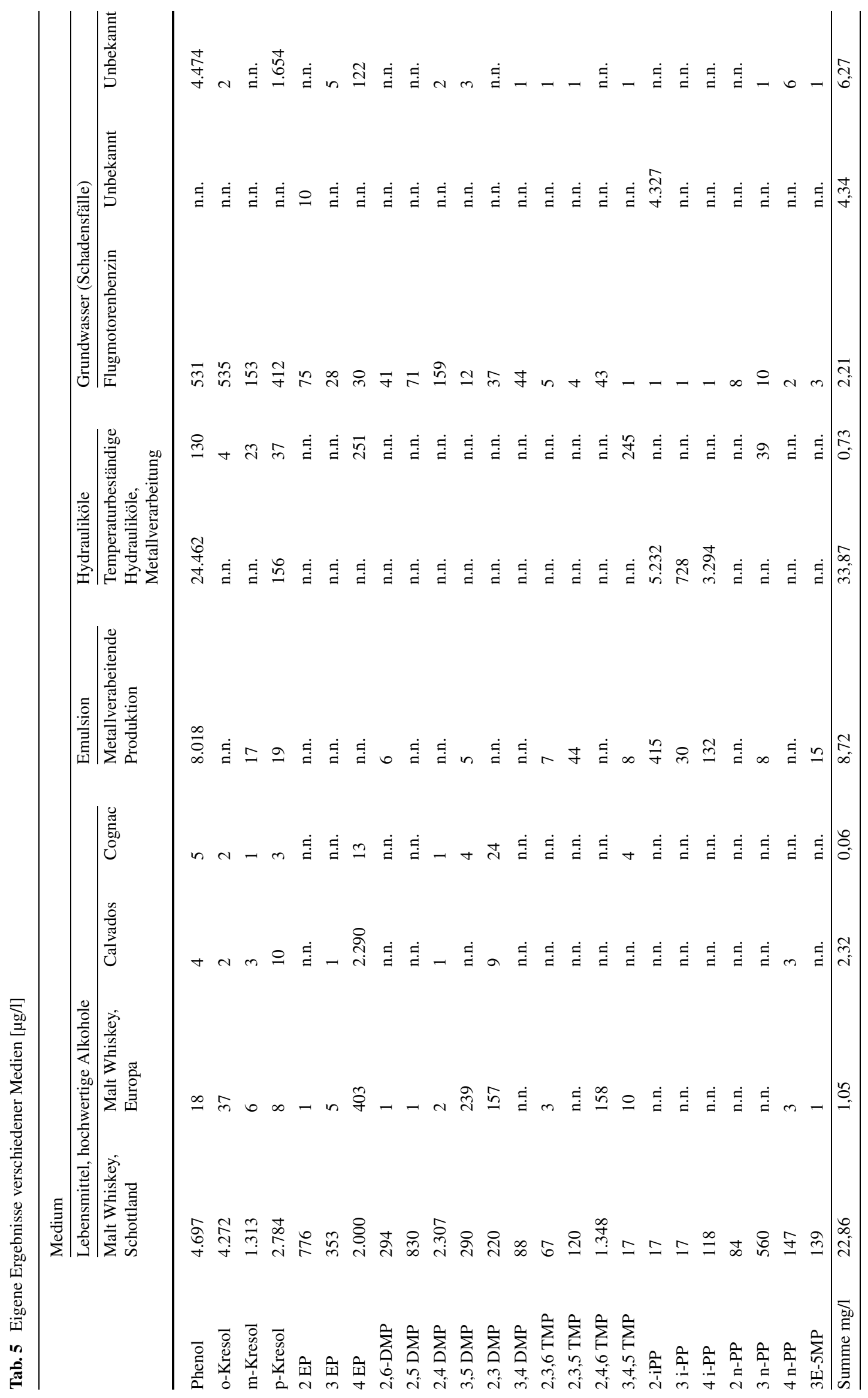




\section{Schlussfolgerungen}

SCAP beschreibt eine Schadstoffgruppe mit 35 hydrophilen, teils persistenten Einzelsubstanzen, deren einzige natürliche Quellen signifikanter Konzentrationen Erdöle bzw. erdölführende Gesteine sind. In verschiedensten Industriezweigen finden sie als einzelne Substanzen oder als Gemische An- und Verwendung, wodurch viele Eintragsquellen in die Umweltmedien möglich sind. Es sind, neben Erdöl, alle weiteren Quellen und Vorkommen kurzkettiger Alkylphenole auf sehr spezifische, industrielle Prozesse zurückzuführen. Die Isomerenverteilungen in Proben dienen als Fingerabdrücke entsprechender Produktionsprozesse und zeigen daher SCAP als hervorragende Fluss- und Prozessindikatoren.

Open Access Dieser Artikel unterliegt den Bedingungen der Creative Commons Attribution License. Dadurch sind die Nutzung, Verteilung und Reproduktion erlaubt, sofern der/die Originalautor/en und die Quelle angegeben sind.

\section{Literatur}

Ahmaruzzaman, M.D.: Adsorption of phenolic compounds on lowcost adsorbents. A review. Adv. Colloid Interface Sci. 143, 48-67 (2008)

Bastow, T.P., Aarssen, B.G.K., Chidlow, G.E., Alexander, R., Kagi, R.I.: Small-scale and rapid quantitative analysis of phenols and carbazoles in sedimentary matter. Org. Geochem. 34, 1113-1127 (2003)

Bennett, B., Bowler, B.F.J., Larter, S.R.: Determination of $\mathrm{C}_{0}-\mathrm{C}_{3}$ alkylphenols in crude oils and water. ACS 68, 3697-3702 (1996)

Bennett, B., Noke, K.J., Bowler, B.F.J., Larter, S.R.: The accurate determination of $\mathrm{C}_{0}-\mathrm{C}_{3}$ alkylphenol concentration in crude oils. J. Environ. Anal. Chem. 87(5), 307-320 (2007)

Böhm, E., Hillenbrand, T., Marscheider-Weidemann, F., Müller, B., Wiederhold, J., Herrchen, M., Klein, M.: Ermittlung der Quellen für die prioritären Stoffe nach Artikel 16 der Wasserrahmenrichtlinie und Abschätzung ihrer Eintragsmengen in die Gewässer in Deutschland. UBA-FB 000394 (2002)

Brenes, M., Romero, C., García, A., Hidalgo, F.J., Ruiz-Méndez, M.V.: Phenolic compounds in olive oils intended for refining: formation of 4-ethylphenol during olive paste storage. J. Agric. Food Chem. 52, 8177-8181 (2004)

Brunner, P.H., Capri, S., Marcomini, A., Giger, W.: Occurrence and behaviour of linear alkylbenzenesulphonates, nonylphenol, nonylphenol mono- and nonylphenol diethoxyylates in sewage and sewage sludge treatment. Water Res. 22, 1465-1472 (1988)

Emsley, J.: Fritten, Fett und Faltencreme: Noch mehr Chemie im Alltag. Wiley-VCH, Weinheim (2009)

Fischer, F., Kerndorff, H., Kühn, S.: Die branchenspezifische Ermittlung von Phenolen und Abschätzung ihrer Grundwassergängigkeit, Bd. 107. WaBoLu e.V., Berlin (2000)

Giger, W., Brunner, P.H., Schaffner, C.H.: 4-Nonylphenol in sewage sludge: accumulation of toxic metabolites from nonionic surfactants. Science 225, 623-625 (1984)

Gundermann, E.: Chemie und Technologie des Braunkohlenteers, 1. Aufl. Akademie Verlag, Berlin (1964)
Ippolo, M., Alexander, R., Kagi, R.I.: Identification of $\mathrm{C}_{0}-\mathrm{C}_{3}$ phenols in some Australian crude oils. Org. Geochem. 18(5), 603-609 (1992)

Isacchi, B., Bergonzi, M.C., Carnevalli, F., van der Esch, S.A., Vincieri, F.F., Bilia, A.R.: Analysis and stability of the constituents of St John's wort oils prepared with different methods. J. Pharm. Biomed. Anal. 45(5), 756-761 (2007)

Kolbe, N.: Analyse von Phenolen in komplexen Matrices nach Derivatisierung zu Ferrocenestern. Dissertationsschrift, Wilhelms-U. Münster (2008)

Kollmansberger, H.: Inhaltsstoffzusammensetzung und sensorische Qualität von 20 Kultivaren verschiedener Capsicum-Arten. Dissertationsschrift, TU München (2007)

Laespada, E.F., Pavón, J.L.P., Cordero, B.M.: Automated on-line membrane extraction liquid chromatographic determination of phenols in crude oils, gasolines and diesel fuels. J. Chromatogr. A 852(2), 395-406 (1999)

Larter, S.R., Bowler, B.F.J., Li, M., Chen, M., Brincat, D., Bennett, B., Noke, K., Donohoe, P., Simmons, D., Kohnen, M., Allan, J., Telnaes, N., Horstad, I.: Molecular indicators of secondary oil migration distances. Nature 383, 593-597 (1996)

Larter, S.R., Gates, I.D., Adams, J.J., Jiang, C., Snowdon, L., Bennett, B., Huang, H.: Preconditioning an oilfield reservoir (BRUTUS). U.S. Full Patent Filed (2007)

Larter, S.R., Bennett, B.: Now you see them, now you don't, now you might see them again! A review of the systematic of alkylphenols occurrence in conventional and heavy oil petroleum systems. Recovery - CSPG CSEG CLWS Convention (2011)

Licha, T., Dermietzel, J., Sauter, M.: SCAP - chemical analysis and field studies. In: Geochemical Processes in Soil and Gruondwater, GeoProc 2002. Forschungsberichte (DFG), Kap. 9, S. 115-130. Wiley-VCH, Weinheim (2003)

Licha, T.: Short chained alkylphenols (SCAP) in groundwaterChemical Analysis, Adsorption Mechanism and Field Cases. Dissertationsschrift, F-S-Universität Jena (2002)

Lo, Y.C., Koziel, J.A., Cai, L., Hoff, S.J., Jenks, W.S., Xin, H.: Simultaneous chemical and sensory characterization of VOCs and semi-VOCs emitted from swine manure using SPME and multidimensional gas chromatography-mass spectrometry-olfactometry system. J. Environ. Qual. 37, 521-534 (2008)

Rayne, S., Eggers, N.J.: 4-ethylphenol and 4-ethylguaiacol in wines: estimating non-microbial sourced contributions and toxicological considerations. J. Environ. Sci. Health. B 42(8), 887-897 (2007)

Shiu, W.-Y., Ma, K.-C., Varhaniczková, D., Mackay, D.: Chlorophenols and alkylphenols: a review and correlation of environmentally relevant properties and fate in an evaluative environment. Chemosphere 29(6), 1155-1224 (1994)

Taylor, P., Larter, S.R., Jones, M., Dale, J., Horstad, I.: The effect of oil-water-rock partitioning on the occurrence of alkylphenols in petroleum systems. Geochim. Cosmochim. Acta 61(9), 18991910 (1997)

Ullmann's Encyklopädie der Technischen Chemie. VCH, Weinheim (1995)

Umweltbundesamt (UBA): Gemeinsamer stoffdatenpool Bund/Länder. www.gsbl.de (02.02.2009)

Varhanickova, D., Shiu, W.Y., Mackay, D.: Aqueous solubilities of alkylphenols and methoxyphenols at $25^{\circ} \mathrm{C}$. J. Chem. Eng. Data 40, 448-451 (1995)

Wasinski, F.A.H.: Ferrocencarbonsäurechlorid als hochselektives Derivatisierungsmittel für Hydroxyverbindungen zur Analyse mittels Gaschromatographie mit Atomemissions- und massenselektivem Detektor (GC-AED und -MSD). Dissertationsschrift, Westfälische Wilhelms-Universität Münster (2004)

Wiedenmann, M.: Hygiene im Rettungsdienst. Elsevier, München (2011) 